Europhysics Letters

PREPRINT

\title{
Consistent particle-based algorithm with a non-ideal equation of state
}

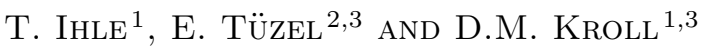 \\ 1 Department of Physics, North Dakota State University, \\ P.O. Box 5566, Fargo, ND 58102, USA. \\ 2 School of Physics and Astronomy, 116 Church Street SE, \\ University of Minnesota, Minneapolis, MN 55455, USA. \\ 3 Supercomputing Institute, University of Minnesota, \\ 599 Walter Library, 117 Pleasant St. SE, Minneapolis, MN 55455, USA
}

PACS. 02.70.Ns - Molecular dynamics and particle methods.

PACS. 47.11.+j - Computational methods in fluid dynamics.

PACS. 05.40.-a - Fluctuation phenomena, random processes, noise, and Brownian motion.

\begin{abstract}
A thermodynamically consistent particle-based model for fluid dynamics with continuous velocities and a non-ideal equation of state is presented. Excluded volume interactions are modeled by means of biased stochastic multiparticle collisions which depend on the local velocities and densities. Momentum and energy are exactly conserved locally. The equation of state is derived and compared to independent measurements of the pressure. Results for the kinematic shear viscosity and self-diffusion constants are presented. A caging and order/disorder transition is observed at high densities and large collision frequency.
\end{abstract}

Introduction. - The efficient modeling of the long length- and time-scale dynamics of complex liquids such as colloidal and polymeric suspensions requires a simplified, coarsegrained description of the solvent degrees of freedom. A recently introduced particle-based simulation technique [1] — often called stochastic rotation dynamics (SRD) [2-7] or multiparticle collision dynamics $[8,9]$ - is a very promising algorithm for mesoscale simulations of this type. In additional to its numerical advantages, the algorithm enables simulations in the microcanonical ensemble, and fully incorporates both thermal fluctuations and hydrodynamic interactions. Furthermore, its simplicity has made it possible to obtain analytic expressions for the transport coefficients which are valid for both large and small mean free paths, something that is often very difficult to do for other mesoscale particle-based algorithms. This algorithm is particularly well suited for studying phenomena with Reynolds and Peclet numbers of order one, and it has been used to study the behavior of polymers $[9,10]$, colloids $[1,11-13]$, vesicles in shear flow [14], and complex fluids $[15,16]$.

The original SRD algorithm models a fluid with an ideal gas equation of state. The fluid is therefore very compressible, and the speed of sound, $c_{s}$, is low. In order to have negligible compressibility effects, as in real liquids, the Mach number has to be kept small, which means

(c) EDP Sciences 
that there are limits on the flow velocity in the simulation. It is therefore important to explore ways to extend the algorithm to model dense fluids. Our approach starts from what has been a common theme of most liquid theories, namely the separation of intermolecular forces into short- and long-range parts, which are then treated differently. The short-range component is a strong repulsion when molecules are close together; it leads to excluded volume effects which cause a decrease in the fluid's compressibility and eventual crystallization at low temperatures or high density. The long-range component is a weak attraction which can lead to a liquidgas transition. The generic reference system for the short-range repulsive component of the force is the hard sphere system. In this letter we show how the SRD the algorithm can be modified to model excluded volume effects, allowing for a more realistic modeling of dense gases and liquids. This is done in a thermodynamically consistent way by introducing generalized excluded volume interactions between the fluid particles. The algorithm can be thought of as a coarse-grained multi-particle collision generalization of a hard sphere fluid, since, just as for hard spheres, there is no internal energy. In order to simplify the analysis of the equation of state and the transport coefficients, and enhance computational efficiency, the cell structure of the original SRD algorithm is retained. This work is a first step towards developing consistent particle-based algorithms for modeling, in the microcanonical ensemble, more general liquids with additional attractive interactions and a liquid-gas phase transition.

Model. - As in the original SRD algorithm, the solvent is modeled by a large number $N$ of point-like particles of mass $m$ which move in continuous space with a continuous distribution of velocities. The system is coarse-grained into $(L / a)^{d}$ cells of a $d$-dimensional cubic lattice of linear dimension $L$ and lattice constant $a$. The algorithm consists of individual streaming and collision steps. In the free-steaming step, the coordinates, $\mathbf{r}_{i}(t)$, of the solvent particles at time $t$ are updated according to $\mathbf{r}_{i}(t+\tau)=\mathbf{r}_{i}(t)+\tau \mathbf{v}_{i}(t)$, where $\mathbf{v}_{i}(t)$ is the velocity of particle $i$ at time $t$ and $\tau$ is the value of the discretized time step. In order to define the collision, we introduce a second grid with sides of length $2 a$ which (in $d=2$ ) groups four adjacent cells into one "supercell".

As discussed in Refs. [2] and [3], a random shift of the particle coordinates before the collision step is required to ensure Galilean invariance. All particles are therefore shifted by the same random vector with components in the interval $[-a, a]$ before the collision step (Because of the supercell structure, this is a larger interval than in the conventional SRD algorithm). Particles are then shifted back by the same amount after the collision. To initiate a collision, pairs of cells in every supercell are randomly selected. As shown in Fig. 1, three different choices are possible: a) horizontal (with $\left.\boldsymbol{\sigma}_{1}=\hat{x}\right)$, b) vertical $\left(\boldsymbol{\sigma}_{2}=\hat{y}\right)$, and c) diagonal collisions (with $\boldsymbol{\sigma}_{3}=(\hat{x}+\hat{y}) / \sqrt{2}$ and $\boldsymbol{\sigma}_{4}=(\hat{x}-\hat{y}) / \sqrt{2}$ ). Note that diagonal collisions are essential to equilibrate the kinetic energies in the $x-$ and $y$-directions.

In every cell, we define the mean particle velocity,

$$
\mathbf{u}_{n}=\frac{1}{M_{n}} \sum_{i=1}^{M_{n}} \mathbf{v}_{i}
$$

where the sum runs over all particles, $M_{n}$, in the cell with index $n$. The projection of the difference of the mean velocities of the selected cell-pairs on $\sigma_{j}, \Delta u=\sigma_{j} \cdot\left(\mathbf{u}_{1}-\mathbf{u}_{2}\right)$, is then used to determine the probability of collision. If $\Delta u<0$, no collision will be performed. For positive $\Delta u$, a collision will occur with an acceptance probability which depends on $\Delta u$ and the number of particles in the two cells, $M_{1}$ and $M_{2}$. This rule mimics a hard-sphere collision on a coarse-grained level: For $\Delta u>0$ clouds of particles collide and exchange momenta. For 


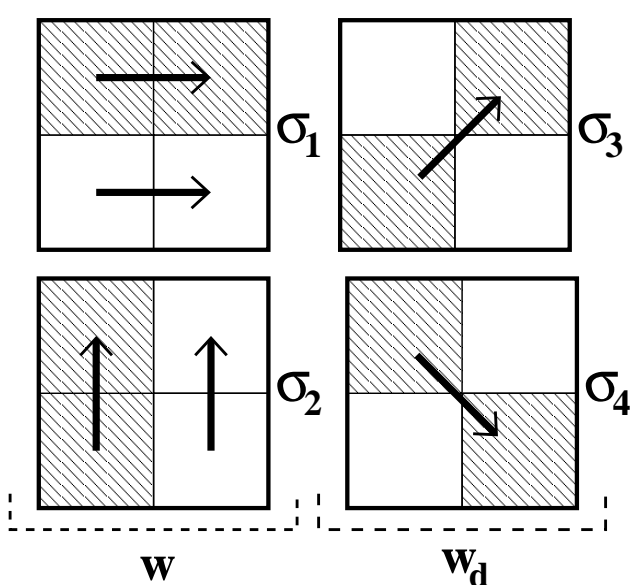

Fig. 1

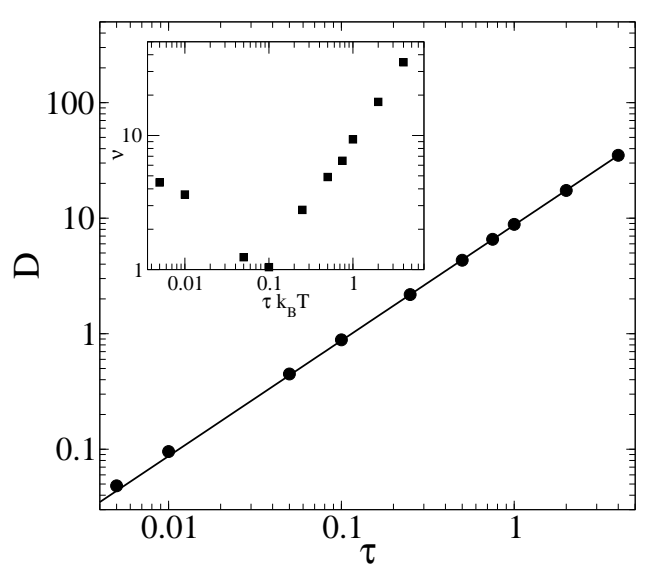

Fig. 2

Fig. 1 - Schematic of collision rules. Momentum is exchanged in four ways, a) horizontally along $\boldsymbol{\sigma}_{1}$, b) vertically along $\boldsymbol{\sigma}_{2}$, c) diagonally and d) off-diagonally along $\boldsymbol{\sigma}_{3}$ and $\boldsymbol{\sigma}_{4}$ respectively, according to Eq. (3). $w$ and $w_{d}$ denote the probabilities of choosing collisions a), b) and c), d) respectively.

Fig. 2 - Diffusion coefficient as a function of $\tau$. The data points in the inset are data for the shear viscosity measured using Green-Kubo relations, as a function of $\tau k_{B} T$. The solid line shows the analytical result, Eq. (9). Parameters: $L=64 a, M=5, k_{B} T=1.0$ and $A=1 / 60$.

reasons discussed in the following, we have used the acceptance probability

$$
p_{A}\left(M_{1}, M_{2}, \Delta u\right)=\Theta(\Delta u) \tanh (\Lambda) \quad \text { with } \quad \Lambda=A \Delta u M_{1} M_{2},
$$

where $\Theta$ is the unit step function and $A$ is a parameter which allows us to tune the equation of state. The hyperbolic tangent function was chosen in (2) in order to obtain a probability which varies smoothly between 0 and 1.

Once it is decided to perform a collision, an explicit form for the momentum transfer between the two cells is needed. The collision should conserve the total momentum and kinetic energy of the cell-pairs participating in the collision, and in analogy to the hard-sphere liquid, the collision should primarily transfer the component of the momentum which is parallel to the connecting vector $\boldsymbol{\sigma}_{j}$. In the following, this component will be called the parallel or longitudinal momentum. There are many different rules which fullfill these conditions. Our goal here is to obtain a large speed of sound. We therefore use a collision rule which leads to the maximum transfer of the parallel component of the momentum and does not change the transverse momentum. The rule is quite simple; it exchanges the parallel component of the mean velocities of the two cells, which is equivalent to a "reflection" of the relative velocities,

$$
v_{i}^{\|}(t+\tau)-u^{\|}=-\left(v_{i}^{\|}(t)-u^{\|}\right)
$$

where $u^{\|}$is the parallel component of the mean velocity of the particles of both cells. The perpendicular component remains unchanged,

$$
v_{i}^{\perp}(t+\tau)=v_{i}^{\perp}(t) .
$$

It is easy to verify that these rules conserve momentum and energy in the cell pairs. 
EUROPHYSICS LETTERS

Because of $x-y$ symmetry, the probabilities for choosing cell pairs in the $x-$ and $y-$ directions (with unit vectors $\boldsymbol{\sigma}_{1}$ and $\boldsymbol{\sigma}_{2}$ in Fig. 1) are equal, and will be denoted by $w$. The probability for choosing diagonal pairs $\left(\boldsymbol{\sigma}_{3}\right.$ and $\boldsymbol{\sigma}_{4}$ in Fig. 1) is given by $w_{d}=1-2 w . w$ and $w_{d}$ must be chosen to that the hydrodynamic equations are isotropic and do not depend on the orientation of the underlying grid. This can be done by considering the temporal evolution of the lowest moments of the velocity distribution function. It is sufficient to consider the following three moments for a single particle $i$,

$$
\Psi_{i}(t)=\left(\begin{array}{c}
\left\langle v_{i x}^{2}(t)\right\rangle \\
\left\langle v_{i y}^{2}(t)\right\rangle \\
\left\langle v_{i x}(t) v_{i y}(t)\right\rangle
\end{array}\right)
$$

Assuming molecular chaos, the collision rules can be used to determine the eigenvalues of the relaxation matrix, $R$, defined by $\Psi_{i}(t+\tau)=R \Psi_{i}(t)$.

Because of the conservation of energy, one of the three eigenvalues of $R$ is equal to one; the other two are given by $\lambda_{1}=w_{d}+2 w(2 / M-1)$ and $\lambda_{2}=2 w+w_{d}(2 / M-1)$, where $M$ is the average number of particles per cell. Isotropy requires that $\lambda_{1}=\lambda_{2}$, a condition that can be fullfiled for arbitrary $M$ only if $w_{d}=1 / 2$ and $w=1 / 4$. Simulations show that both the speed of sound and the shear viscosity are isotropic for this choice. Note, however, that this does not guarantee that all properties of the model are isotropic. This becomes apparent at high densities or high collision frequency, $1 / \tau \gg 1$, where inhomogenuous states with cubic or rectangular order can be observed (see Fig. 4 and accompanying discussion).

Transport coefficients. - The transport coefficients can be determined using the same Green-Kubo formalism as was used for the original SRD algorithm [2-4]. In particular, the kinematic shear viscosity is given by

$$
\nu=\frac{\tau}{N k_{B} T} \sum_{n=0}^{\infty}\left\langle S_{x y}(0) S_{x y}(n \tau)\right\rangle,
$$

where

$$
S_{x y}(n \tau)=\sum_{j=1}^{N}\left(v_{j x}(n \tau) \Delta \xi_{j y}(n \tau)+\Delta v_{j x}(n \tau)\left[\Delta \xi_{j y}^{s}(n \tau)-z_{j l y}^{s}([n+1] \tau) / 2\right]\right)
$$

is the off-diagonal element of the stress tensor $\mathbf{S} . \boldsymbol{\xi}_{j}(t)$ and $\boldsymbol{\xi}_{j}^{s}(t)$ are the cell coordinates of particle $j$ in the fixed and shifted frames at time $t$, respectively, $\Delta \boldsymbol{\xi}_{j}(t)=\boldsymbol{\xi}_{j}(t+\tau)-\boldsymbol{\xi}_{j}(t)$, $\Delta \boldsymbol{\xi}_{j}^{s}(t)=\boldsymbol{\xi}_{j}(t+\tau)-\boldsymbol{\xi}_{j}^{s}(t+\tau)$, and $\Delta \mathbf{v}_{j}(t)=\mathbf{v}_{j}(t+\tau)-\mathbf{v}_{j}(t)$. $\mathbf{z}_{j l}^{s}$ indexes pairs of cells which participate in a collision event; the second subscript, $l$, is the index of the collision vectors $\boldsymbol{\sigma}_{l}$ listed in Fig. 1. For example, for collisions characterized by $\boldsymbol{\sigma}_{1}, z_{j 1 x}^{s}=1$ if $\xi_{j x}^{s}$ in (7) is one of the two cells on the left of a supercell and $z_{j 1 x}^{s}=-1$ if $\xi_{j x}^{s}$ is on the right hand side of a supercell; all other components of $\mathbf{z}^{s}$ are zero. In general, the components of $\mathbf{z}_{j l}^{s}$ are either 0 , 1 , or -1 . Using $\left\{\mathbf{z}_{j l}^{s}\right\}$, the collision invariants of the model can be written as

$$
\sum_{j}\left(\mathrm{e}^{i \mathbf{k} \cdot \xi_{j}^{s}(t+\tau)}+\mathrm{e}^{i \mathbf{k} \cdot\left(\xi_{j}^{s}(t+\tau)+\mathbf{z}_{j l}^{s}(t+\tau)\right)}\right)\left[a_{\beta, j}(t+\tau)-a_{\beta, j}(t)\right]=0,
$$

where $a_{1, j}=1$ for the density, $\left\{a_{\beta, j}\right\}=\left\{v_{\beta-1, j}\right\}$ are components of the particle momentum, and $a_{d+2, j}=v_{j}^{2} / 2$ is the kinetic energy of particle $\mathrm{j}$ [3]. The analogous collision invariants for the standard SRD algorithm are given in Eq. (25) of [3]. The vectors $\mathbf{z}^{s}$ are constructed so 
that the sum of the two exponentials in (8) is the same for two particles if and only if they are in partner cells in a collision with index $l$ (see Fig. 1).

The self-diffusion constant $D$ is given by a sum over the velocity-autocorrelation function (see, e.g. Eq. (102) in [3]) and can be evaluated analytically assuming molecular chaos. Due to the excluded volume interactions, density fluctuations are supressed in the current algorithm; ignoring these fluctuations, one finds

$$
D=k_{B} T \tau\left(\frac{1}{A} \sqrt{\frac{\pi}{k_{B} T}} \frac{M^{-3 / 2}}{1+1 /(8 M)}-\frac{1}{2}\right),
$$

which is in good agreement with simulation data, see Fig. 2.

Equation of state. - The collision rules conserve the kinetic energy, so that the internal energy of our system should be the same as that of an ideal gas. Thermodynamic consistency requires that the non-ideal contribution to the pressure is linear in $T$. As will be shown, this is possible if the coefficient $A$ in (2) is chosen small enough (see Fig. 3).

We use here the mechanical definition of pressure - the average longitudinal momentum transfer across a fixed interface per unit time and unit surface area - to determine the equation of state. We consider only the momentum transfer due to collisions, since that coming from streaming constitutes the ideal part of the pressure.

Take an interface that is parallel to the $y$-axis and consider the component $p_{x x}$ of the pressure tensor. Only collisions with label $l=1,3$, and 4 of the collision vector $\boldsymbol{\sigma}_{l}$ in Fig. 1 contribute to the momentum transfer in this case. Consider the contribution to the momentum transfer across the cell boundary from collisions with $l=1$. For fixed number of particles, $M_{1}$ and $M_{2}$, in the two cells, the thermal average of the momentum transfer, $\Delta G_{x}$, across the dividing line is

$$
\left\langle\Delta G_{x}\right\rangle=\frac{w}{2} \int_{0}^{\infty} p_{G}(\Delta u) p_{A}\left(M_{1}, M_{2}, \Delta u\right) \Delta G_{x} d(\Delta u) .
$$

The factor $1 / 2$ comes from the position average of the dividing line, since the collision occurs $\mathrm{n}$ the shifted cells, and the integral is restricted to positive $\Delta u$ because the acceptance rate is zero for $\Delta u<0$. $p_{G}(\Delta u)$ is the probability that $u_{1 x}-u_{2 x}$ for the micro-state of two cells is equal to $\Delta u$. $w=1 / 4$ is the probability of selecting this collision.

Expanding the acceptance probability, Eq. (2), in $\Lambda \equiv A \Delta u M_{1} M_{2}$ leads to $p_{A}\left(M_{1}, M_{2}, \Delta u\right)=\Theta(\Delta u)\left(\Lambda-\Lambda^{3} / 3+\ldots\right)$. The contributions to the pressure from all terms of this series can be calculated, but since the resulting contribution to the pressure from a term proportional to $\Lambda^{n}$ is of order $T^{n}$, we restrict ourselves to the first term.

The resulting contribution to the pressure, $P\left(\sigma_{1}, M_{1}, M_{2}\right)$, for fixed $M_{1}$ and $M_{2}$ is the average momentum transfer per unit area and unit time, so that using Eqs. (10), we have $P\left(\boldsymbol{\sigma}_{1}, M_{1}, M_{2}\right)=w A k_{B} T M_{1} M_{2} /(2 a \tau)+O\left(A^{3} T^{2}\right)$. A similar calculation can be performed for the contributions from the diagonal collisions, which occur with the probability $w_{d}$. Using $w=1 / 4$ and $w_{d}=1 / 2$ and averaging over the number of particles per cell (assuming that they are Poisson-distributed and that the particle number distributions in adjacent cells are not correlated), one finds the non-ideal part of the pressure,

$$
P_{n}=P_{i d}\left(\frac{1}{2 \sqrt{2}}+\frac{1}{4}\right) \frac{A M}{2} \frac{a}{\tau}+O\left(A^{3} T^{2}\right) .
$$

where $P_{i d}=k_{B} T M / a^{2}$ is the ideal gas contribution to the pressure (in $\left.d=2\right)$. Note that the same result is obtained if, instead of averaging over $M_{1}$ and $M_{2}$, we simply set $M_{1}=M_{2}=M$, the average number of particles per cell. $P_{n}$ is quadratic in the particle density, $\rho=M / a^{2}$, 


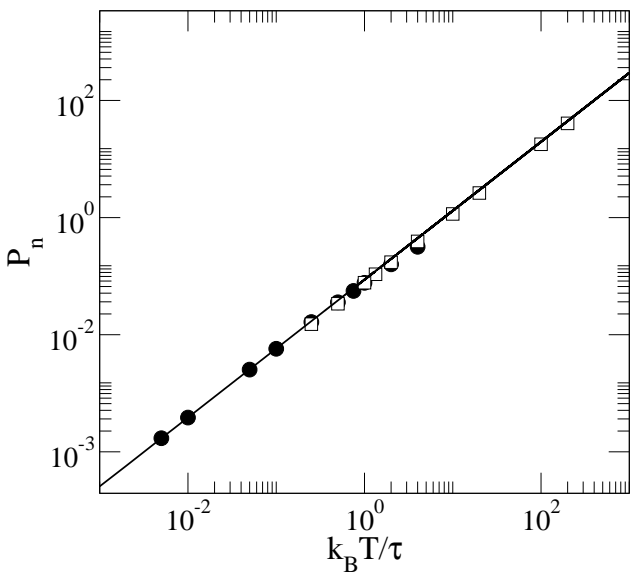

Fig. 3

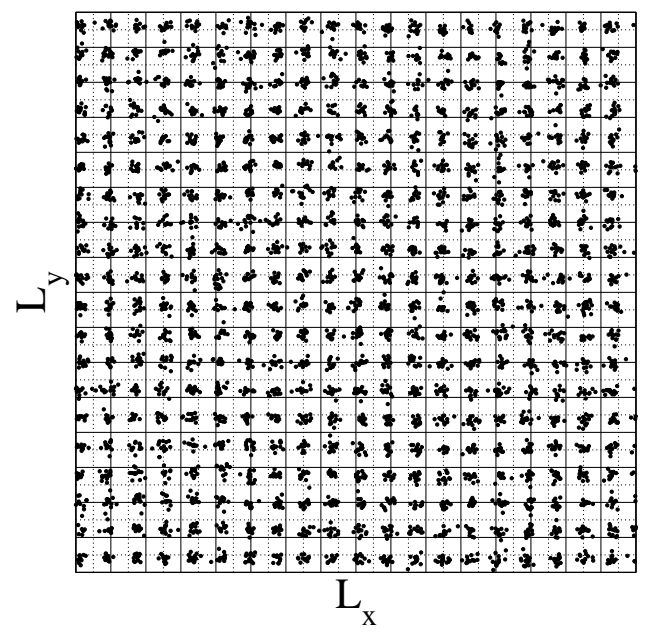

Fig. 4

Fig. 3 - Non-ideal pressure, $P_{n}$, as a function of $k_{B} T / \tau$ averaged over $10^{5}$ time steps. Both $k_{B} T$ and $\tau$ ranged from 0.005 to 4 . The line represents the theoretical expression, Eq. (11). For $\tau=0.005$ and $k_{B} T=1, P_{n}$ is five times larger than $P_{i d}$. Parameters: $L=64 a, M=5, A=1 / 60$.

Fig. 4 - Freezing snapshot after $10^{6}$ time steps. Parameters: $L_{x}=L_{y}=32 a, M=5, k_{B} T=$ $3.125 \times 10^{-5}, \tau=10^{-3}, A=1 / 60$.

as one would expect from a virial expansion. The prefactor $A$ must be chosen small enough that higher order terms in this expansion are negligible. We have found that prefactors $A$ leading to acceptance rates of about $20 \%$ are sufficiently small to guarantee that the pressure is linear in $T$ (see Fig. 3). In order to measure $P_{n}$, we have used the fact that the average of the diagonal part of the microscopic stress tensor gives the virial expression for the pressure

$$
P=P_{i d}+P_{n}=\left\langle\sum_{j}\left\{v_{j x} \Delta \xi_{j x}+\Delta v_{j x}\left[\Delta \xi_{j x}^{s}-z_{j l x}^{s} / 2\right]\right\}\right\rangle .
$$

The first term, $\left\langle v_{x, j} \Delta \xi_{x, j}\right\rangle=\left\langle\tau v_{x, j}^{2}\right\rangle$, gives $P_{i d}$, as discussed in Ref. [3]. The average over the second term vanishes (see Ref. [3]), while the average of the third term is the non-ideal part of the pressure, $P_{n}$. Simulation results for $P_{n}$ obtained using (12) are in good agreement with the analytical expression, (11) (see Fig. 31). In addition, measurements of the density fluctuations, $\left\langle\left|\rho_{k}\right|^{2}\right\rangle$, at small wave vectors $\mathbf{k}$, as well as results for the adiabatic speed of sound obtained from simulations of the dynamic structure factor, are both in good agreement with the predictions following from Eq. (11). These results provide strong evidence for the thermodynamic consistency of the model.

Caging and order/disorder transition. - If the non-ideal part of the pressure is large compared to the ideal pressure, ordering effects can be expected. For small $A$, both contributions to the pressure are proportional to the temperature, so that just as in a real hard-sphere fluid, changing the temperature does not lead to an order/disorder transition. On the other hand, the two contributions to the pressure have different dependencies on the density and time step, $\tau$. In fact, $\tau$ can be interpreted as a parameter describing the efficiency of the collisions; lowering $\tau$ results in a higher collision frequency, and has a similar effect to making 
the spheres larger in a real hard-sphere system. We therefore expect caging and ordering effect if either $\rho$ is increased or $\tau$ is decreased. This is indeed the case. For $\tau<0.0016, \rho=5$ and $k_{B} T=1.0$, an ordered cubic state is observed. The cubic symmetry of the ordered state is clearly an artifact of the cubic cell structure, and it would be interesting to see if this could be removed by using an hexagonal cell structure or incorporating random rotations of the grid. One of the surprising features of this crystalline-like state is, that $x-y$ symmetry can be broken. Furthermore, there is the possibility of having several metastable crystalline states corresponding to slightly different lattice constants and number of particles per "cloud". As expected, the lattice constants of these ordered states are slightly smaller than the super-cell spacing, $2 a$, which sets the range of the multi-particle interaction. In this state, the diffusion coefficient becomes very small; particles are caged and can barely leave their location.

To understand this behavior, note that without collisions, particle clouds will broaden due to streaming; this will happen faster the higher the temperature. Due to the grid shift, particles at the perimeter of the clouds will more often undergo collisions with neighbor clouds. These collisions backscatter the particles, forcing them to fly back towards the center of their cloud. There is a correlation between the distance from cloud center and rate at which it is backscattered, leading to stable cloud formation. A particle which is left alone between clouds will feel repulsion from all clouds and moves around very quickly until it is absorbed into a cloud.

Conclusion. - The model presented in this letter is the first extension of the SRD algorithm to model fluids with a non-ideal equation of state. It was shown that the model is thermodynamically consistent for the correct choice of acceptance probabilities and reproduces the correct isotropic hydrodynamic equations at large length scales. Expressions for the equation of state and the self-diffusion constant were derived and shown to be in good agreement with numerical results. Simulation results for the kinematic viscosity were presented, and it was shown that there is an ordered state for large densities and collision frequencies. A detailed analysis of the transport coefficients will be presented elsewhere.

Acknowledgement. - Support from the National Science Foundation under Grant No. DMR-0513393 and ND EPSCoR through NSF grant EPS-0132289 are gratefully acknowledged. We thank A.J. Wagner for numerous discussions.

\section{REFERENCES}

[1] A. Malevanets and R. Kapral, J. Chem. Phys. 110, 8605 (1999); 112, 7260 (2000).

[2] T. Ihle and D.M. Kroll, Phys. Rev. E 63, 020201(R) (2001).

[3] T. Ihle and D.M. Kroll, Phys. Rev. E 67, 066705 (2003); Phys. Rev. E 67, 066706 (2003).

[4] T. Ihle, E. Tüzel, D.M. Kroll, Phys. Rev. E 70, 035701(R) (2004).

[5] E. Tüzel, M. Strauss, T. Ihle and D.M. Kroll, Phys. Rev. E 68, 036701 (2003).

[6] N. Kikuchi, C.M. Pooley, J.F. Ryder, J.M. Yeomans, J. Chem. Phys. 119, 6388 (2003).

[7] C.M. Pooley and J.M. Yeomans, J. Phys. Chem. B (2005).

[8] A. Lamura G. Gompper, T. Ihle and D.M. Kroll, Europhys. Lett. 56, 319 (2001).

[9] M. Ripoll, K. Mussawisade, R.G. Winkler, and G. Gompper, Europhys. Lett. 68, 106 (2004).

[10] N. Kikuchi, A. Gent, and Y. Yeomans, European Phys. J. E 9, 63 (2002).

[11] S.H. Lee and R. Kapral, J. Chem. Phys. 121, 11163 (2004).

[12] J.T. Padding and A.A. Louis, Phys. Rev. Lett. 93, 220601 (2004).

[13] M. Hecht, J. Harting, T. Ihle, and H.J. Herrmann, Phys. Rev. E 78, 011408 (2005).

[14] H. Noguchi and G. Gompper, Phys. Rev. Lett. 93, 258102 (2004).

[15] Y. Hashimoto, Y. Chen, and H. Ohashi, Comp. Phys. Commun. 129, 56 (2000).

[16] T. Sakai, Y. Chen, and H. Ohashi, Phys. Rev. E 65, 031503 (2002). 